\title{
COMPRESSIVE SENSING IN NONSTATIONARY ARRAY PROCESSING USING BILINEAR TRANSFORMS
}

\author{
Yimin D. Zhang and Moeness G. Amin \\ Center for Advanced Communications, Villanova University, Villanova, PA 19085, USA
}

\begin{abstract}
Compressive sensing (CS) has successfully been applied to reconstruct sparse signals and images from few observations. For multi-component nonstationary signals characterized by instantaneous frequency laws, the sparsity exhibits itself in the time-frequency domain as well as the ambiguity domain. In this paper, we examine CS in the context of nonstationary array processing. We show that the spatial averaging of the ambiguity function across the array improves the CS performance by reducing both noise and cross-terms. The corresponding time-frequency distribution which is reconstructed through $L_{1}$ minimizations yields significant improvement in time-frequency signature localizations and characterizations.
\end{abstract}

\section{INTRODUCTION}

Compressive sensing (CS) has successfully been applied to electromagnetic (EM) sensing problems using multiple antennas, including synthetic aperture radar (SAR) and indoor imaging $[1,2,3,4,5]$. In this paper, we apply CS to sparse signal time-frequency representation using antenna arrays. We consider nonstationary signals with instantaneous frequency (IF) characterization. These signals are encountered in many applications including radar, sonar, audio, and biomedicine. The spatial degrees-of-freedom, offered by the multi-sensor system configuration, are used to reduce noise and cross-terms in the ambiguity domain without altering the signal sparsity profile in the joint-variable bilinear representation. This leads to higher time-frequency signature resolution when applying sparse signal reconstruction techniques.

In bilinear time-frequency analyses of nonstationary signals, the existence of cross-terms is a major problem that compromises, and even obscures, the proper identification of signal energy distribution in the time-frequency domain. To mitigate the effect of cross-terms, it is desirable to design a mask that only captures the auto-term components in the ambiguity domain. This concept underlines interferencereducing kernels in the model-free Cohen's class of timefrequency distributions [6]. While many time-frequency kernels, such as the well-known Choi-Williams distribution [7], are data-independent, others, such as the adaptive optimal kernel, optimize the kernel based on the signal characteristics [8]. In either case, cross-term mitigation is achieved at the expense of reduced resolution in the time-frequency

This work is supported in part by the Office of Naval Research (ONR) under Grant N00014-11-1-0576. domain. In multi-sensor systems, on the other hand, it is shown in $[9,10]$ that the averaging of the time-frequency distributions, each is computed at a single sensor, amounts to spatial filtering of the cross-terms. The level of cross-term reduction depends on the spatial correlation between the corresponding source signals. Mathematically, averaging the time-frequency distributions across the array amounts to taking the trace of the spatial time-frequency distribution (STFD) matrix [11, 12].

In this paper, we examine quadratic time-frequency analysis of nonstationary signals in a CS multi-sensor array platform. The focus is on proper identifications of auto-term regions in the ambiguity domain which is key to accurate sparse signal reconstruction. We first show that, because the ambiguity function and the time-frequency distribution are related by the two-dimensional (2-D) Fourier transform, spatial averaging of the ambiguity functions correspond to the same averaging operation in the time-frequency domain, both lead to cross-term and noise reductions. This sensor averaging operation, combined with lowpass filtering in ambiguity domain, which emphasizes the signal autoterms, enhances the signal-to-interference-plus-noise ratio (SINR) without altering the sparsity profile of the signal joint-variable representation. Compressive sensing performed on the weighted/ masked ambiguity function observations yields improved time-frequency signal energy localizations. This improvement is witnessed over single-sensor based approaches as well as when compared to performing $L_{2}$ norm minimization and 2-D Fourier transform.

The following notations are used in this paper. A lower (upper) case bold letter denotes a vector (matrix). $(\cdot)^{*},(\cdot)^{T}$ and $(\cdot)^{H}$ respectively denote complex conjugation, transpose, and conjugate transpose (Hermitian) operations. $\mathbf{I}_{n}$ expresses the $n \times n$ identity matrix. $\mathcal{F}(\cdot)$ and $\mathcal{F}^{-1}(\cdot)$ respectively represent the 2-D Fourier transform and 2-D inverse Fourier transform. $\|\cdot\|_{1}$ and $\|\cdot\|_{2}$ respectively denote the $L_{1}$ and $L_{2}$ norm operations. $\dot{\phi}(t)=d \phi(t) / d t$ represents the derivative of $\phi(t)$ with respect to time. In addition, $\mathbb{C}^{N \times M}$ denotes the complete set of $N \times M$ complex entries.

\section{SIGNAL MODEL}

Consider $K$ narrowband nonstationary signals impinging on an array consisting of $N$ sensors. The $N \times 1$ received data vector $\mathbf{y}(t)$ and the $K \times 1$ source signal vector $\mathbf{d}(t)$ are related by

$$
\mathbf{y}(t)=\mathbf{H d}(t)+\mathbf{n}(t)
$$

where $t$ is the time index, $\mathbf{H}=\left[\mathbf{h}_{1}, \mathbf{h}_{2}, \ldots, \mathbf{h}_{K}\right] \in \mathbb{C}^{N \times K}$ is the mixing matrix that holds the steering vectors of the $K$ 
signals, and $\mathbf{h}_{q}$ is the spatial signature for the $q$ th source, $d_{q}(t)$, that may arrive with or without an angular bearing. $T$ is the number of observation samples. Each element of $\mathbf{d}(t)=\left[d_{1}(t), d_{2}(t), \ldots, d_{K}(t)\right]^{T}$ is assumed to be a monocomponent signal. $\mathbf{n}(t) \in \mathbb{C}^{N \times 1}$ is an additive noise vector that consists of independent and identically distributed (i.i.d.) zero-mean, white and complex Gaussian distributed processes with variance $\sigma_{n}^{2} \mathbf{I}_{N}$. The noise elements are assumed to be independent of the signals, which are assumed to be deterministic.

\section{TIME-FREQUENCY DISTRIBUTION AND AMBIGUITY FUNCTION}

For a nonstationary signal $x(t)$, its ambiguity function is defined as

$$
A_{x x}(\theta, \tau)=\int_{-\infty}^{\infty} x\left(u+\frac{\tau}{2}\right) x^{*}\left(u-\frac{\tau}{2}\right) e^{-j \theta \tau} d u
$$

where $\theta$ and $\tau$ are the frequency lag and the time lag, respectively. Similarly, the time-frequency distribution, within the Cohen's class, is defined as the 2-D Fourier transform of the ambiguity function [6]

$$
D_{x x}(t, f)=\int_{-\infty}^{\infty} \int_{-\infty}^{\infty} \phi(\theta, \tau) A_{x x}(\theta, \tau) e^{-j 2 \pi \theta t-j 2 \pi f \tau} d \theta d \tau,
$$

where $t$ and $f$ represent the time and frequency indexes, respectively, and $\phi(\theta, \tau)$ is the time-frequency kernel. A timefrequency kernel is often designed to emphasize auto-terms and mitigate cross-terms interference. A kernel is typically a 2-D low-pass filter in the ambiguity domain. The reasoning behind this filter is that the auto-terms are generally concentrated around the origin, whereas the cross-terms are positioned away from the origin. However, the exact autoand cross-term distributions depends on the waveforms and thus may differ significantly for different signals.

For two nonstationary signals $x_{i}(t)$ and $x_{k}(t)$, their crossterm ambiguity function can be defined as

$$
A_{x_{i} x_{k}}(\theta, \tau)=\int_{-\infty}^{\infty} x_{i}\left(u+\frac{\tau}{2}\right) x_{k}^{*}\left(u-\frac{\tau}{2}\right) e^{-j \theta \tau} d u
$$

and their cross-term time-frequency distribution is defined as

$D_{x_{i} x_{k}}(t, f)=\int_{-\infty}^{\infty} \int_{-\infty}^{\infty} \phi(\theta, \tau) A_{x_{i} x_{k}}(\theta, \tau) e^{-j 2 \pi \theta t-j 2 \pi f \tau} d \theta d \tau$.

An element of vector $\mathbf{y}(t)$ in (1) is a mixture of multiple signal arrivals and therefore becomes a multi-component signal. Therefore, its ambiguity function and time-frequency distribution, due to their bilinear nature, contain auto-terms and cross-terms. The cross-terms often obscure the identification of the auto-term regions, which reveal the true signal power distribution and, as such, the underlying nonstationary signal characteristics. Cross-term mitigation or reduction is also important for sparse signal reconstruction, as shown in the simulation section.

\section{SPATIAL AVERAGING}

The ambiguity function and time-frequency distribution are two different representations that map a time-domain nonstationary signal to 2-D joint-variable representations that allow more flexible processing and filtering. The proper selection of signal auto-terms amounts to signal-to-noise ratio (SNR) enhancement and enables source signature discriminations. The latter provides improved direction-ofarrival estimation, source separation, and waveform recovery $[13,14]$.

For most commonly used time-frequency kernels, the auto-terms time-frequency distributions are real. These terms are also positive for meaningful time-frequency points where the signal energy is concentrated. On the other hand, cross-terms are complex in general, and their values depend on the relative phase between the contributing signals. As such, the simple averaging of time-frequency distributions over different antennas enhances the auto-terms. The same averaging operation reduces cross-terms if the spatial correlation between the contributing signals is low.

With the focus on cross-term suppression, we consider a noise-free scenario, where the $i$ th diagonal element of $\mathbf{D}_{\mathbf{y y}}(t, f)$ is expressed as $[10]$

$$
D_{y_{i} y_{i}}(t, f)=\sum_{l=1}^{K} \sum_{k=1}^{K} a_{i l} a_{i k}^{*} D_{d_{l} d_{k}}(t, f),
$$

where $a_{i l}$ is the $(i, l)$ th element of A. Averaging the $N$ diagonal elements of $\mathbf{D}_{\mathbf{y y}}(t, f)$ thus becomes

$$
\bar{D}_{\mathbf{y y}}(t, f)=\frac{1}{N} \sum_{i=1}^{N} D_{y_{i} y_{i}}(t, f)=\sum_{l=1}^{K} \sum_{k=1}^{K} \beta_{l, k} D_{d_{l} d_{k}}(t, f),
$$

where $D_{d_{l} d_{k}}(t, f)$ denotes the cross-term between signal component $d_{l}(t)$ and $d_{k}(t)$, and

$$
\beta_{l, k}=\frac{1}{N} \sum_{i}^{N} a_{i l} a_{i k}^{*}
$$

is the spatial correlation, defined in the $N$-element array, of the return signals from targets $l$ and $k$.

The ambiguity function, whether in the form of autoterms or cross-terms, is in general complex. However, the auto-term of a signal component is invariant with the phase difference across the sensor array due to differences in the relative propagation delays. On the other hand, different signal components originated from different sources will yield different phase delays due to differences in their angular locations. Therefore, spatial averaging of the ambiguity functions bears the same spatial filtering capability for cross-term reduction as the averaging of time-frequency distributions. This can be easily inferred because the 2-D Fourier transform associating the ambiguity function and time-frequency distribution is linear. As a result, the ambiguity function averaged over the $N$ array sensors can be expressed as

$$
\bar{A}_{\mathbf{y y}}(\theta, \tau)=\frac{1}{N} \sum_{i=1}^{N} A_{y_{i} y_{i}}(\theta, \tau)=\sum_{l=1}^{K} \sum_{k=1}^{K} \beta_{l, k} A_{d_{l} d_{k}}(\theta, \tau),
$$


where $A_{d_{l} d_{k}}(\theta, \tau)$ is the cross-term ambiguity function between $d_{l}(t)$ and $d_{k}(t)$.

\section{CS-BASED RECONSTRUCTION OF TIME-FREQUENCY REPRESENTATION}

Consider that each signal source is a single-component AMFM signal:

$$
d_{i}(t)=a_{i}(t) e^{j \phi_{i}(t)}, \quad i=1, \ldots, K,
$$

where $a_{i}(t)$ and $\phi_{i}(t)$ are, respectively, the amplitude and phase of the $i$ th signal.

Ideally, its time-frequency distribution should represent the total energy along time-frequency trajectories according to

$$
D_{d_{i} d_{i}}(t, f)=a_{i}^{2}(t) \delta\left(f-\dot{\phi}_{i}(t) /(2 \pi)\right) .
$$

In reality, however, the resolution of time-frequency distributions is highly limited by a number of factors, such as the length of available data and the applied window. This is further complicated with multiple signal arrivals. The CS-based approach seeks a perfectly localized solution that minimizes the total number of nonzero coefficients, i.e., the $l_{0}$-norm of the time-frequency distribution. Consider $\mathbf{D}$ as a time-frequency distribution matrix defined over a proper $(t, f)$ region, and $\mathbf{A}$ the corresponding ambiguity function over a proper ambiguity function domain. Then, the desired localized time-frequency is represented as the solution of the constrained minimization problem [15]:

$$
\mathbf{D}=\arg \min _{\mathbf{B}}\|\mathbf{B}\|_{1} \quad \text { s.t. } \quad \mathcal{F}^{-1}(\mathbf{B})-\mathbf{A}=0 .
$$

The primary constraint which is given by the above expression imposes a strict equality over $\Omega$ in the ambiguity domain. This however can be relaxed according to [16]

$$
\mathbf{D}=\arg \min _{\mathbf{B}}\|\mathbf{B}\|_{1} \quad \text { s.t. }\left\|\mathcal{F}^{-1}(\mathbf{B})-\mathbf{A}\right\|_{2} \leq \epsilon,
$$

where $\epsilon$ is a user-specific bound. Using results from convex optimization, it is shown that the above problem can be related to the basis pursuit denoising (BPDN) [17]:

$$
\mathbf{D}=\arg \min _{\mathbf{B}} \lambda\|\mathbf{B}\|_{1}+\frac{1}{2}\left\|\mathcal{F}^{-1}(\mathbf{B})-\mathbf{A}\right\|_{2},
$$

where $\lambda \geq 0$ is the Lagrange multiplier. In this paper, we form $\mathbf{A}$ from $\bar{A}_{\mathbf{y y}}(\theta, \tau)$ over a properly masked ambiguity region $\Omega$ and perform the above CS $L_{1}$ norm minimization to reconstruct a time-frequency distribution estimate $\mathbf{D}$. The results are compared to others obtained from WignerVille distribution and from the 2-D inverse Fourier transform of masked ambiguity functions. In the proposed technique, the spatial averaging of ambiguity function results in enhanced auto-terms and reduced cross-terms, whereas the CS approach yields highly localized time-frequency energy distributions.

\section{SIMULATION RESULTS}

Consider a uniform linear array consisting of 4-elements with a half-wavelength interelement spacing. Two nonstationary signals impinge on the array with their respective arbitrary spatial signatures that are randomly generated with independent complex Gaussian distribution with zero mean and unit variance:

$$
\begin{aligned}
& \mathbf{H}^{T}= \\
& {\left[\begin{array}{rrrr}
-1.22+0.24 j & -1.39+0.66 j & 0.16-0.39 j & 0.28-0.39 j \\
1.13+0.95 j & -0.44-0.35 j & -0.50-0.26 j & 1.13-0.33 j
\end{array}\right] .}
\end{aligned}
$$

The spatial correlation matrix of the mixing matrix is given by

$$
\mathbf{H}^{H} \mathbf{H}=\left[\begin{array}{cc}
4.33 & -0.28-0.55 j \\
-0.28+0.55 j & 4.20
\end{array}\right]
$$

The signals emitted from the two sources are linear FM signals with closely separated signatures. Their IF laws are expressed as

$$
f_{1}(t)=0.05+0.005 t, \quad f_{2}(t)=0.07+0.005 t,
$$

where $t=0,1, \ldots, 79$. The transmitted signals as well as the noise are assumed to have a unit power.

Fig. 1(a) shows the Wigner-Ville distribution of the signal received at the first sensor. Due to the close signatures of the two source signals and the presence of the crossterms, the two signals are not clearly separated. Fig. 1(b) shows the Wigner-Ville distribution averaged over the four sensors. Because of the low spatial correlation between the two sources, the cross-terms are significantly suppressed, leading to better auto-term identification. Similarly, Fig. 2(a) shows the ambiguity function of the signal received at the first sensor, and Fig. 2(b) shows the results averaged over the four sensors. Cross-terms are observed in Fig. 2(a) that are parallel to the auto-terms, whereas they almost disappear in Fig. 2(b).

Fig. 3(a) shows the masked results of the spatially averaged ambiguity function. We use a simple rule such that an ambiguity function region with its magnitude below $10 \%$ of the peak value is discarded from selection. The timefrequency distribution reconstructed from the CS approach is shown in Fig. 3(b), where $\lambda$ is chosen as 0.01. It clearly shows high-resolution auto-terms and reduced noise and cross-terms for improved time-frequency signature localizations and characterizations.

For comparison, Fig. 4(a) shows the time-frequency distribution obtained using $L_{2}$-norm reconstruction. The results are similar to those shown in Fig. 1(b) but with reduced noise in general. Fig. 4(b) shows the CS-based timefrequency distribution reconstructed from the single-sensor observation at the first antenna. To achieve the similar sparsity and noise reduction, the ambiguity domain mask is chosen to discard ambiguity regions with magnitude below $15 \%$ of the peak value. Note that due to the close separation, it is difficult to discriminate cross-terms from the auto-terms in the single-sensor ambiguity function. As the result, while achieving noise reduction, strong cross-terms remain in the reconstructed time-frequency representation.

\section{CONCLUSION}

We have used the time, frequency, and space variables associated with nonstationary array processing to improve sparse signal signature reconstruction and characterizations. Spatial averaging of the ambiguity functions across the array reduces cross-terms, while maintaining the same sparsity profile of the joint-variable signal representation. This 
allows the compressive sensing (CS) to act on data of much higher signal-to-interference-plus-noise ratio (SINR). It was shown that this pre-processing step leads to finer resolution and more accurate representation of the nonstationary signal behavior when solving the $L_{1}$ norm minimization problem in the time-frequency domain.

\section{REFERENCES}

[1] R. Baraniuk and P. Steeghs, "Compressive radar imaging," in Proc. IEEE Radar Conf., Waltham, MA, pp. 128-133, April 2007.

[2] M. Herman and T. Strohmer, "High-resolution radar via compressive sensing," IEEE Trans. Signal Process., vol. 57, no. 6, pp. 2275-2284, 2009.

[3] Y. Yoon and M. G. Amin, "Compressed sensing technique for high-resolution radar imaging," in Proc. SPIE, vol. 6968, pp. $69681 \mathrm{~A}-69681 \mathrm{~A}-10,2008$.

[4] J. Moulton, S. A. Kassam, F. Ahmad, M. G. Amin, and K. Yemelyanov, "Target and change detection in synthetic aperture radar sensing of urban structures," in Proc. IEEE Radar Conf., Rome, Italy, May 2008.

[5] M. G. Amin, F. Ahmad, W. Zhang, "A compressive sensing approach to moving target indication for urban sensing, inProc. IEEE Radar Conf., Kansas City, MO, May 2011.

[6] L. Cohen, "Time-frequency distributions - a review," Proc. IEEE, vol. 77, no. 7, pp. 941-981, July 1989.

[7] H. I. Choi and W. J. Williams, "Improved time-frequency representation of multicomponent signals using exponential kernels," IEEE Trans. Acoust., Speech, Sig. Proc., vol. ASSP-37, no. 6, pp. 862-871, June 1989.

[8] D. L. Jones and R. G. Baraniuk, "An adaptive optimal-kernel time-frequency representationm," IEEE Trans. Signal Proc., vol. 43, no. 10, pp. 2361-2371, Oct. 1995.

[9] L. Cirillo, A. M. Zoubir, N. Ma, and M. Amin, "Automatic classification of auto- and cross-terms of time-frequency distributions in antenna arrays," in Proc. IEEE ICASSP, Orlando, FL, May 2002.

[10] W. Mu, M. G. Amin, and Y. Zhang, "Bilinear signal synthesis in array processing," IEEE Trans. Signal Proc., vol. 51, no. 1, pp. 90-100, Jan. 2003.

[11] N. Linh-Trung, A. Belouchrani, K. Abed-Meraim, and B. Boashash, "Separating more sources than sensors using timefrequency distributions," EURASIP J. Applied Signal Proc., vol. 2005, no. 17, pp. 2828-2847, 2005.

[12] Y. Zhang and M. G. Amin, "Blind separation of nonstationary sources based on spatial time-frequency distributions", EURASIP J. Applied Signal Proc., vol. 2006, article ID 64785, 13 pages, 2006.

[13] M. G. Amin, A. Belouchrani, and Y. Zhang, "The spatial ambiguity function and its applications," IEEE Signal Proc. Lett., vol. 7, no. 6, pp. 138-141, June 2000.

[14] Y. Zhang, W. Mu, and M. G. Amin, "Subspace analysis of spatial time-frequency distribution matrices," IEEE Trans. Signal Proc., vol. 49, no. 4, pp. 747-759, April 2001.

[15] P. Flandrin and Pierre Borgnat, "Time-frequency energy distributions meet compressed sensing," IEEE Trans. Signal Proc., vol. 58, no. 6, pp. 2974-2982, June 2010.

[16] E. Candès and T. Tao, "Near-optimal signal recovery from random projections: Universal encoding strategies?" IEEE Trans. Inf. Theory, vol. 52, no. 12, pp. 5406-5425, 2006.

[17] S. S. Chen, D. L. Donoho, and M. A. Saunders, "Atomic decomposition by basis pursuit," SIAM J. Sci. Comput., vol. 20, pp. pp. 33-61, 2001.

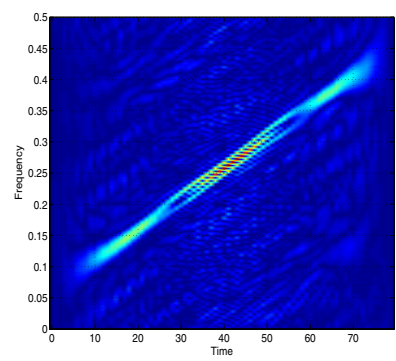

(a) From a single sensor

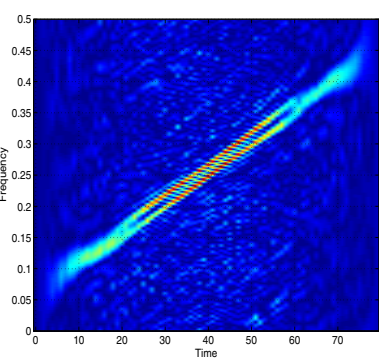

(b) Spatially averaged
Fig. 1 Wigner-Ville distribution.

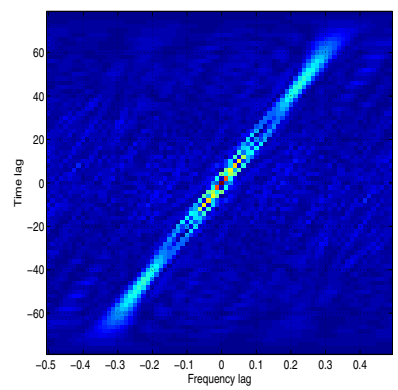

(a) From a single sensor

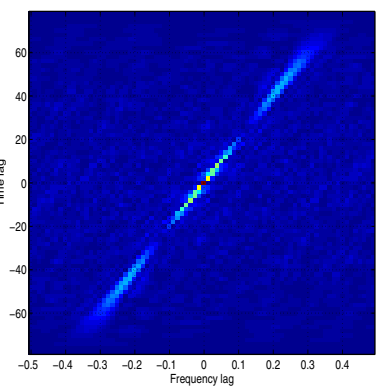

(b) Spatially averaged
Fig. 2 Ambiguity function.

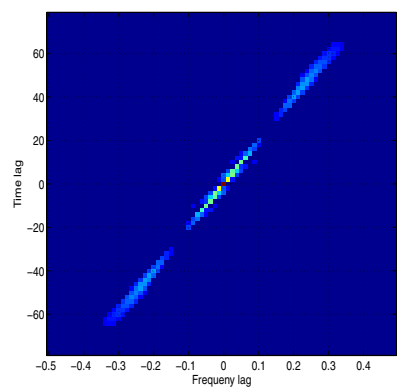

(a) Masked ambiguity function

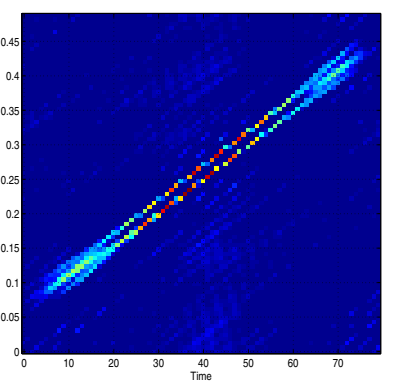

(b) CS-based result
Fig. 3 Reconstruction of time-frequency distribution through CS.

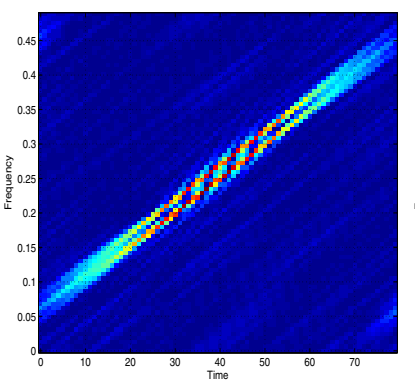

(a) Via $L_{2}$-norm approach

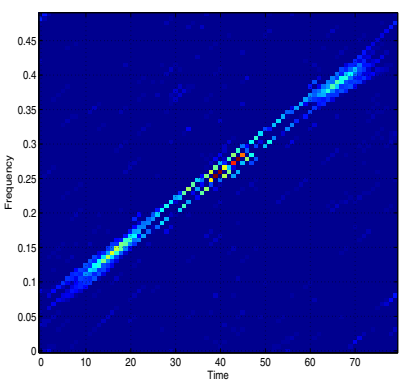

(b) From single-sensor data
Fig. 4 Time-frequency distributions for comparison. 